\title{
Implementación de la estrategia de servitización en las empresas manufactureras es- pañolas
}

\author{
Helen Castellón-Orozco, Natalia Jaría-Chacón, Laura Guitart-Tarrés
}

Recibido: 09 de Septiembre de 2019

https://doi.org/10.37610/dyo.v0i70.568

Aceptado: 19 de Noviembre de 2019

\section{Resumen}

La elevada competencia a la que se enfrentan las empresas hoy en día obliga a las organizaciones a establecer nuevas estrategias de diferenciación. De ello no se escapan las empresas manufactureras. En este sentido, las empresas manufactureras están añadiendo nuevas prestaciones adicionales a sus productos, tradicionalmente desarrolladas por empresas de servicios. La apuesta por complementar un producto fabricado con servicios adicionales que añadan valor (servitización) es una buena alternativa para conseguir la tan preciada diferenciación en las empresas manufactureras.

A pesar de ello, hoy en día es todavía escasa la investigación que ha indagado empíricamente sobre la servitización que resulta ser una estrategia competitiva y rentable para un fabricante. Por este motivo, esta investigación analiza cómo la implementación de la estrategia de servitización afecta al rendimiento empresarial, es decir, cómo los resultados de la empresa se ven afectados por el hecho de que las empresas estén o no servitizadas.

A partir del análisis de una base de datos de mil empresas manufactureras españolas y, tras el tratamiento estadístico realizado, se demuestra que las empresas altamente servitizadas obtienen mejores resultados empresariales que aquellas con un bajo nivel de implementación de esta estrategia. Con ello se concluye el efecto positivo, aunque no proporcional, que la oferta de servicios ejerce en los ingresos de las empresas y se demuestra que las compañías con un alto grado de servitización obtienen mejores resultados empresariales que aquellas con un bajo nivel de implementación.

\section{Palabras clave}

Servitización, implementación, empresas manufactureras, servicio.

\section{Introducción}

Hoy en día en el ámbito empresarial, el aumento de la demanda, las presiones competitivas y la búsqueda de mejores resultados llevan a las empresas manufactureras a hacer un esfuerzo adicional para aumentar el valor de sus productos. En esta línea, la estrategia de servitización, conocida como el proceso de creación de valor añadiendo servicios a los productos (Vandermerwe y Rada, 1988), justamente persigue este objetivo de diferenciar los productos que ofrece una compañía.

Helen Castellón-Orozco *
hcasteor11@alumnes.ub.edu
Natalia Jaría-Chacón **
nataliajaria@ub.edu
Laura Guitart-Tarrés ***
laura.guitart@ub.edu
* Estudiante de PhD. Departamento de Empresa. Universi
dad de Barcelona, España
$* *$ Profesora agregada del Departamento de Empresa. Uni
versidad de Barcelona, España
$* * *$ Profesora titular del Departamento de Empresa. Univer-
sidad de Barcelona, España

El término servitización se mencionó por primera vez a finales de 1980 y desde entonces, ha tomado relevancia para los investigadores, especialmente en industrias manufactureras (Neely, 2008). Esta estrategia fortalece las relaciones con los clientes, crea nuevos flujos de ingresos y establece una barrera de entrada para los competidores (Baines et al., 2009 y 2011).

Tradicionalmente añadir valor para un fabricante se basaba únicamente en la transformación de determinados inputs en unos outputs. Sin embargo, el cliente actual empieza a valorar otros servicios adicionales que pueden complementar este producto final. Por ello, las empresas estrictamente manufactureras deben adaptarse para satisfacer estas nuevas necesidades de los clientes, siendo una oportunidad para ellas abrirse a una nueva concepción que acoja un mayor nivel de desarrollo de los servicios. La servitización trata de aportar este complemento adicional de servicios, ampliando el valor añadido y extendiéndose tanto a los productos físicos como a los servicios complementarios.

Bajo esta idea, el proceso de servitización deja de ser exclusivo de las empresas de servicios, puesto que las empresas manufactureras están ya combinando sus productos físicos con otros servicios asociados, agregando valor a sus 
productos (Arias-Aranda y Jaría-Chacón, 2014). De hecho, las empresas son ya plenamente conscientes de que la oferta y la prestación de esos servicios suele requerir de un diseño y una estrategia inicial más complejos que la propia fabricación tradicional de productos. Ello implica asumir que las ópticas deben de ser diferentes y las empresas de fabricación tradicionales deben pensar ahora también en clave de servicios.

En la actualidad, la servitización ya no es una estrategia de negocio alternativa, sino que se ha convertido en una estrategia recurrente para la supervivencia (Slepniov et al., 2010). Las empresas manufactureras se sienten atraídas principalmente por la servitización como un medio para generar beneficios y fortalecer la relación con el cliente (Baines et al., 2009). Pero para aprovechar estas potenciales oportunidades, las empresas necesitan establecer una alineación apropiada entre las condiciones del mercado y las características de su organización. Para que las empresas tradicionales de fabricación puedan sobrevivir en un mercado global y hacer frente a la elevada competencia con la que coexisten, la estrategia de servitización les ofrece mejorar su competitividad y obtener ventajas competitivas frente al resto de empresas del sector (Gebauer et al., 2008). Asimismo, las necesidades del cliente cada vez más amplias y con demandas integrales en mercados cada vez más maduros, obligan a los empresarios a ser constantemente innovadores con el objetivo de diferenciar sus productos y conservar su rentabilidad.

Aunque existe una vasta literatura que analiza cualitativamente la servitización, solamente algunos autores han estudiado la relación empírica existente entre la servitización y la rentabilidad de la empresa, como Visnjic Kastalli y Van Looy (2013), Fang et al. (2008) y Han et al. (2013) entre otros. En el trabajo de Visnjic Kastalli y Van Looy (2013) miden cuantitativamente la relación existente de la servitización (medida según los ingresos procedentes de los servicios) sobre la no servitización (medida a través de los ingresos obtenidos por productos puramente tradicionales). Otros estudios realizan un análisis sobre la servitización en relación al tipo de servicio ofrecido (Li et al., 2015), así como con el número de servicios ofrecidos (Neely, 2008) y también en base a nuevos productos servitizados ofrecidos (Falk, 2014). Estas investigaciones concluyen que la relación entre la servitización y los resultados de la empresa se ve también afectada por otros factores, tales como el grado de innovación en el producto, la complejidad del mismo, las áreas de negocio, el diseño de la organización y el nivel de inversión aplicado. Algunos estudios, como el de Malleret (2006), señalan asimismo que la oferta de servicios no siempre garantiza resultados rentables debido a que la estrategia adecuada del servicio depende también en gran medida del entorno de gestión.

Parece lógico pensar que el grado de servitización en una empresa afecta a todo el conjunto de la organización, por lo tanto, la adopción de una estrategia de este tipo requiere también de la adquisición de nuevos recursos y capacidades
(Martínez et al., 2010; Neu y Brown, 2005). Debido a esto, es preciso considerar que los empleados necesitan una amplia base de conocimientos técnicos, además de poseer las competencias conductuales apropiadas. Es decir, las estrategias de recursos humanos deben estar diseñadas para seleccionar, potenciar y retener a los empleados que posean algunas características indispensables.

En este sentido, el estudio de Fiksdal y Kumar (2011) sobre empresas manufactureras noruegas concluye que estas compañías están experimentando una creciente competencia respecto de las empresas afincadas en países en vía de desarrollo, las cuales presentan unos costes de producción notablemente más bajos. Este aumento de la competencia obliga a los fabricantes a diferenciar y agregar más valor a su oferta, con el fin de mantener su competitividad y no perder clientela. Ello supone un ejemplo más de cómo la estrategia de servitización representa una interesante vía alternativa, ya que mediante la expansión del modelo de negocio y la inclusión de los servicios, los fabricantes tradicionales llegan a ofrecer soluciones cada vez más valoradas por los clientes.

En consecuencia, siendo la servitización una estrategia en alza, cada vez más utilizada y, al mismo tiempo poco analizada empíricamente con detalle, este trabajo profundiza en su estudio y aporta al debate para medir cuantitativamente su efecto. Concretamente, el objetivo de este artículo es analizar si la adopción de la estrategia de servitización en las empresas españolas contribuye en el rendimiento empresarial de la industria manufacturera para el año 2016.

Finalmente, este articulo muestra las particularidades de la aplicación de los servicios en empresas recomendando cuando es rentable su implementación en diversos tipos de industrias manufactureras.

\section{Estado del Arte}

La estrategia de servicio se define, en general, por cómo las empresas deben diferenciarse de sus competidores por medio de las ofertas de servicios. El estudio de Gebauer et al. (2010) argumenta que el establecimiento de una estrategia de servicio clara es un importante factor de éxito, permitiendo altos ingresos en concepto de servicio en las empresas de fabricación. De hecho, una estrategia de servicio claramente definida animará a las empresas a adoptar las medidas organizativas apropiadas y la asignación de recursos más conveniente. Además, el desarrollo de nuevas ideas de servicio en servicio-productos altamente aceptados también requiere de un proceso de servicio-desarrollo claramente determinado (Gebauer et al., 2006).

Las empresas analizadas en el estudio de Gebauer et al. (2010) percibieron que una estrategia de servicio exitosa no puede desarrollarse sin la participación de todas las áreas relevantes de una empresa y, por lo tanto, debe de haber una aceptación prácticamente generalizada de la estrategia y 
contar con el compromiso de los departamentos clave del negocio. A su vez, estos mismos investigadores dedujeron también la importancia que tienen los procedimientos (análisis de la estrategia, desarrollo, implementación y monitoreo), los cuales deben ser sistemáticos, transparentes y que incorporen ciclos de retroalimentación frecuentes (Gebauer et al., 2010).

Otros autores consideran que la comercialización activa de los servicios puede aumentar sustancialmente los ingresos y que puede considerarse como una vía para el éxito económico (Potts, 1988; Reinartz y Ulaga, 2008). En la mayoría de las ocasiones, de hecho, las motivaciones económicas son el centro de atención de los fabricantes que apuestan por el cambio de enfoque corporativo de pasar de productos a servicios. Además, los directivos que buscan aumentar los ingresos de servicio en las empresas manufactureras tienen que aprender una forma completamente nueva de hacer negocios.

En esta misma línea, Homburg et al. (2003), Oliva y Kallenberg (2003) y Gebauer et al. (2012) establecen que los servicios aumentan la rentabilidad global de las empresas manufactureras, ya que los ingresos por servicios presentan mayores márgenes de beneficio que los generados por los productos. También Baines et al. (2007), Davies (2004), Nordin et al. (2011) y Salonen (2011) concluyen que las empresas industriales se esfuerzan cada vez más por complementar sus productos mediante la adición de servicios. La idea clave es que productos y servicios ofertados de manera conjunta forman un tándem de mayor valor añadido que consigue integrar un paquete de soluciones muy valorado por el cliente $y$, por consiguiente, acaba dando altos márgenes de beneficio y permite conseguir unos ingresos más estables (Brady et al., 2005; Davies et al., 2007; Gebauer y Fleisch, 2007; Kohtamäki et al., 2013; Kowalkowski, 2010; Meier et al., 2011).

Investigadores como Gebauer et al. (2010) o Fischer et al. (2010) también apuestan por la fórmula según la cual la prestación de servicios en las empresas manufactureras es capaz de conseguir ingresos más estables que si la empresa se centrara únicamente en la oferta de productos. Esto sucede así porque los servicios tienden a generar ingresos durante todo el ciclo de vida del producto, incluso en los periodos de recesión económica cuando las ventas del producto se ven afectadas.

En consecuencia, los servicios pueden ser la vía para ofrecer una nueva fuente sostenible de ingresos para el fabricante, ayudando a: 1) superar el estancamiento de los mercados de productos tradicionales (Eggert et al., 2011; Slack, 2005; Gebauer y Fleisch 2007); 2) mejorar el crecimiento de ingresos y beneficios (Eggert et al., 2014); 3) proporcionar un flujo más estable de ingresos (Wise y Baumgartner, 1999); 4) aumentar la rentabilidad (Gebauer et al., 2012; Kohtamäki et al., 2013; Dachs et al., 2014); 5) crear fuentes diversas para obtener ingresos (Baines y
Lightfoot, 2013); 6) conseguir ofrecer una mejora en las respuestas a las necesidades del cliente (Ostrom et al., 2010); 7) ayudar en la apuesta por una continua innovación de productos (Eggert et al., 2011); y, 8) establecer mayores barreras de entrada a la competencia (Oliva y Kallenberg, 2003).

En cuanto a las ventajas de adoptar una óptica de servitización en una empresa manufacturera, Li et al. (2015) destacan las siguientes. La primera de ellas es que, debido a la característica de no duplicidad de los servicios, una empresa puede mejorar la satisfacción y la lealtad del cliente a través de la oferta de servicios únicos y sin precedentes, fortaleciendo así la competitividad de la empresa. La segunda ventaja es que los ingresos de una empresa pueden incrementarse a través de servicios prestados mediante el uso de un conocimiento profesional acumulado. Éste es un caso típico en el que el conocimiento se transforma en beneficios económicos, ya que las ofertas de servicios basadas en el conocimiento promueven la venta de productos y, en última instancia, impulsan el desarrollo de la empresa. Y la tercera ventaja es que como los recursos son siempre limitados con precios fluctuantes, el hecho de añadir servicios en la cartera de la empresa implica que ésta ya no dependa sólo de productos como fuente de beneficios, por lo que la servitización puede reducir la dependencia sobre los recursos con el fin de minimizar el impacto de esta situación, y, por consiguiente, sobre su rentabilidad.

A pesar de que las ventajas y consecuencias de una estrategia de servitización parecen evidentes y atractivas, por el momento, sólo algunos estudios confirman el efecto positivo de los servicios en el desempeño empresarial de las empresas industriales (Davies, 2004; Oliva y Kallenberg, 2003). Sin embargo, estudios recientes son menos concluyentes sobre el nivel exacto de rendimiento que los servicios industriales generan, e incluso sugieren una influencia negativa en ciertos puntos (Neely, 2008) o un efecto de rendimiento no lineal (Fang et al., 2008). En general, estas conclusiones contradictorias hacen pensar que la relación entre los servicios industriales y los resultados de una empresa es mucho más compleja de lo que ha sido anticipada por estudios empíricos previos.

Aunque pueda parecer extensa la literatura existente en esta área, los estudios fundamentados en bases de datos no son tan frecuentes y los resultados no son claros (De la calle y Freije, 2016). La paradoja de la servitización (Neely et al., 2011) se refleja en el debate sobre sus efectos económico-financieros. Mientras algunos autores presentan evidencias de los beneficios de la servitización, basados habitualmente en el estudio de casos concretos, trabajos más recientes indican que en la práctica, la complejidad de los retos de la implementación de la estrategia puede incluso provocar caídas de resultados. Los estudios que valoran la servitización desde una perspectiva más cuantitativa, confirman que los servicios no son por sí mismos garantía de rápidos resultados y que el cuidado de la implementación de la estrategia es imprescindible. Aunque los beneficios de la servitización parecen 
muy claros, en realidad, sólo algunas de las empresas manufactureras están implementando una estrategia intensiva de alto nivel, entre las cuales, las que tienen éxito son aún menos (Li et al., 2015).

La transformación de las empresas manufactureras hacia un modelo de servitización es más que una innovación del modelo de negocio, es un cambio en el patrón económico-empresarial. Esta tendencia se debe a la demanda imperativa de las empresas para mejorar y mantener la competitividad y adaptarse a un mercado cada vez más agresivo y en el que es difícil sobrevivir. Sin embargo, muchos fabricantes no están recogiendo los resultados esperados o, en otras palabras, no han tenido éxito en explotar toda la capacidad en el rendimiento empresarial.

El éxito competitivo o rendimiento empresarial se refiere al beneficio económico que ofrece cada unidad productiva en una empresa, se puede medir en términos de mejoras en el rendimiento, como el retorno de la inversión, la participación en el mercado (Porter 1985). Así mismo se utilizan otras medidas para medir el rendimiento empresarial: más específicamente la rentabilidad de la empresa sobre las ventas (ROS) (Neely, 2008 ; Suárez et al., 2013 ; Visnji et al., 2016 ), los ingresos de la empresa (Rianartz y Ulaga, 2008; Maier et al., 2011; Kohtamaki et al., 2013), el EBIT (Valtakoski y Witell, 2018) y el EBITDA (Visintin y Rapaccini, 2010).

Sin embargo, existe poca evidencia sistemática y sólida del impacto de la servitización en el desempeño de la empresa. Además, el análisis de este impacto sigue siendo controvertido. Investigaciones anteriores han demostrado que la mayoría de los beneficios esperados de la servitización (en términos de mayores ingresos o mayor rentabilidad, por ejemplo) no se materializa en muchos casos, dando lugar a la denominada "paradoja del servicio" (Gebauer et al., 2005 ). Además, la mayoría de la evidencia empírica disponible se basa en estudios de casos a nivel de empresa o muestras limitadas de empresas relativamente grandes.

\section{Metodología}

\subsection{Recogida y descripción de los datos}

Además de la poca evidencia empírica sobre la implementación de la estrategia de servitización, la literatura plantea también una paradoja en los servicios en cuanto a que parece más difícil para las empresas obtener beneficios mediante la adición de los servicios (Gebauer et al., 2005; Reinartz y Ulaga 2008). Para abordar estos temas, este artículo analiza cómo la implementación de la servitización en las empresas manufactureras españolas afecta al rendimiento empresarial.

Para alcanzar este objetivo, esta investigación utiliza una base de datos de empresas manufactureras españolas sobre la que se realiza un análisis cuantitativo. Los datos del estu- dio se extraen de la base de datos SABI (Sistema Ibérico de Análisis de Balances) que contiene los valores financieros de 2 millones de empresas españolas y más de 500.000 de compañías portuguesas. El año de referencia es el 2016, dado que es el último año con datos completos de la información necesaria para el análisis en el momento de inicio del análisis empírico.

En la metodología de selección de los datos, se utiliza como referencia el trabajo de Neely (2008), cuyo principal objetivo es estudiar el alcance y la magnitud de la servitización a partir de una muestra de 10.028 empresas manufactureras de 25 países diferentes. Tomando como base este estudio, primero se realiza la clasificación inicial de las empresas manufactureras de SABI, a partir de los códigos SIC (Standard Industrial Classification) primarios o secundarios entre el rango de valores de 10 a 39 inclusive (Tabla 1). Esta selección inicial permite identificar 1.456.709 empresas manufactureras españolas.

A continuación, se seleccionan las empresas de mayor dimensión (medidas en términos de ingresos y número de empleados, teniendo en cuenta aquellas con un volumen de ingresos con un mínimo de 50.000 euros y un número mínimo de 50 empleados), ya que son éstas las que pueden representar un mejor exponente de implementación de la estrategia de servitización (Neely, 2008); obteniendo una muestra de mil empresas. En este sentido, trabajos como el de Neely (2008) muestran que las empresas más grandes, medidas tanto en términos de número de empleados como de ingresos, tienden a servitizar más que las empresas más pequeñas. También Kwak y Kim (2016) concluyen que el tamaño de las empresas ayuda a observar su efecto en el rendimiento de las empresas.

El siguiente paso consiste en la clasificación de las empresas que implantan la estrategia de servitización, la cual se basa en las características clave que define la literatura sobre este fenómeno. En este sentido, Neely (2008) propone categorizar los servicios en las empresas manufactureras según los doce siguientes tipos: diseño y desarrollo de servicios, sistemas y soluciones, comercio al por menor y distribución, mantenimiento y soporte, instalación e implementación, servicios financieros, propiedad y bienes raíces, consultoría, outsourcing y servicios operativos, servicios de compras, servicios de arrendamiento, transporte y servicios de transporte de mercancías. Este trabajo considera que las empresas servitizadas son las que ofrecen al menos uno de estos servicios tipificados por Neely (2008), de manera que las empresas que no poseían alguno de ellos se consideraron en la categoría de "no servitizadas".

Posteriormente, bajo estas características teóricas, se realiza un proceso de codificación manual de cada empresa una a una para la clasificación de mil empresas seleccionadas a partir de SABI en las categorías de servitizadas o no servitizadas según las categorías de Neely (2008), y contabilizando la cantidad de servicios ofrecidos y establecer el 
nivel de servitización. Este proceso se efectúa a partir de la información disponible en la página web corporativa de cada empresa, valorando el número y el tipo de servicios ofrecidos. Al final del análisis de las mil empresas manufactureras españolas, 73 fueron eliminadas al no tener las condiciones para ser codificadas. Dicha codificación se efectúa a partir de la información disponible en la página web corporativa de cada empresa filtrando los servicios que ofrecen cada empresa, y a partir de la información contenida en la base de datos FACTIVA se verificaron los datos de cada empresa respecto a SABI. Esta base de datos FACTIVA es una herramienta de investigación e información de negocios, propiedad de Dow Jones \& Company. Se trata de la fuente líder mundial de noticias, datos e ideas sobre compañías, que ayuda a los profesionales en la toma de decisiones empresariales a través de su potente búsqueda de información sobre empresas.
El proceso de clasificación finaliza en una muestra final de 927 empresas (al descartar 73 de ellas por falta de información en sus páginas web).

La Tabla 1 recoge un primer análisis descriptivo de las características de las empresas manufactureras de la muestra obtenida. Se observa que un $41 \%$ de las empresas españolas analizadas (380 de las 927 compañías) sí servitizan, es decir, ofrecen al menos uno de los servicios establecidos por Neely (2008).

Por otro lado, también se puede apreciar el nivel de implementación de la estrategia de servitización en función del sector de actividad, ya que la columna de porcentaje (\%) de servitización de la Tabla 1 recoge el número de empresas con al menos un servicio.
Tabla 1 Implementación de la servitización en las empresas manufactureras españolas. Fuente: elaboración propia

\begin{tabular}{|c|c|c|c|}
\hline Código & $\begin{array}{c}\text { Sistema de clasificación internacional } \\
\text { (SIC) }\end{array}$ & Número de empresas & \% Servitización \\
\hline 13 & Petróleo y gas natural & 8 & 100 \\
\hline 35 & Maquinaria & 30 & 90 \\
\hline 37 & Equipos de transporte & 105 & 80 \\
\hline 17 & Contratistas especializados & 33 & 78.79 \\
\hline 38 & $\begin{array}{l}\text { Instrumentos de medida, análisis, control, } \\
\text { fotografía, óptica y relojes }\end{array}$ & 13 & 69.23 \\
\hline 25 & Mueble y mobiliario & 3 & 66.67 \\
\hline 29 & Petróleo y sus derivados & 3 & 66.67 \\
\hline 16 & Construcciones pesadas y contratas & 24 & 58.33 \\
\hline 34 & $\begin{array}{l}\text { Fabricación de metal excepto maquinaria y } \\
\text { equipos transporte }\end{array}$ & 37 & 56.76 \\
\hline 36 & Maquinaria eléctrica y electrónica & 41 & 56.10 \\
\hline 22 & Industria textil & 4 & 50 \\
\hline 30 & Productos de goma y plástico & 21 & 47.62 \\
\hline 15 & Construcciones de obras y contratas & 41 & 43.90 \\
\hline 14 & $\begin{array}{l}\text { Minería y explotación de yacimientos min- } \\
\text { erales no metálicos }\end{array}$ & 8 & 37.50 \\
\hline 32 & $\begin{array}{l}\text { Productos de piedra, arcilla, vidrio y hor- } \\
\text { migón }\end{array}$ & 35 & 37.14 \\
\hline 28 & Productos químicos & 147 & 36.73 \\
\hline 33 & Siderurgia & 58 & 36.21 \\
\hline 39 & Fabricantes diversos & 3 & 33.33 \\
\hline
\end{tabular}




\begin{tabular}{clcc}
\hline 10 & Minerales metálicos & 5 & 20 \\
\hline 26 & Papel y derivados & 39 & 15.38 \\
\hline 20 & Industria alimentaria & 228 & 14.47 \\
\hline 27 & Editorial - artes gráficas & 18 & 11.11 \\
\hline 21 & Carbón (lignito y hulla) & 1 & 0 \\
\hline 23 & Fabricantes de tabaco & 5 & 0 \\
\hline 24 & Prendas confeccionadas & 12 & 0 \\
\hline 31 & Industria de la madera & 3 & 0 \\
\hline Total & Cuero y derivados & 2 & $\mathbf{4 0 . 9 9}$ \\
\hline
\end{tabular}

Un segundo análisis de la muestra permite establecer qué servicios son los más ofertados por las compañías españolas servitizadas. En este sentido, la Tabla 2 muestra que los servicios más comúnmente ofrecidos por las empresas de fabricación son: mantenimiento y soporte $(60.53 \%)$, seguido de diseño y desarrollo (10.79\%) y comercio al por menor y distribución (10.26\%). Ello puede justificarse dado que estos servicios representan un soporte imprescindible para aquellos productos con características técnicas más complejas, que deben ser ofrecidos por las propias empresas que los han diseñado y desarrollado.
Tabla 2 Tipología de servicios ofrecidos por las empresas servitizadas. Fuente: elaboración propia

\begin{tabular}{|c|c|c|}
\hline Tipo de servicio & Número de empresas & $\%$ de empresas que ofrecen el servici \\
\hline Mantenimiento y soporte & 230 & $60.53 \%$ \\
\hline Diseño y desarrollo & 41 & $10.79 \%$ \\
\hline Comercio al por menor y distribución & 39 & $10.26 \%$ \\
\hline Sistemas y soluciones & 29 & $7.63 \%$ \\
\hline Servicios de compras & 11 & $2.89 \%$ \\
\hline Servicio de consultoría & 10 & $2.63 \%$ \\
\hline Instalación e implementación & 9 & $2.37 \%$ \\
\hline $\begin{array}{l}\text { Transporte y servicios de transporte de } \\
\text { mercancías }\end{array}$ & 8 & $2.11 \%$ \\
\hline Servicios financieros & 2 & $0.53 \%$ \\
\hline Propiedad y bienes raíces & 1 & $0.26 \%$ \\
\hline Total & 380 & $100 \%$ \\
\hline
\end{tabular}


En cuanto al número de servicios que ofrecen las empresas servitizadas, como se puede observar en la siguiente tabla, lo más común por el momento es que las empresas ofrezcan un solo servicio, pero es cierto que poco a poco va tomando fuerza la oferta de más servicios. De esta manera, el $41 \%$ resultante de servitización en las empresas analizadas se distribuye de la siguiente manera:
Tabla 3 Cantidad de servicios que ofrecen las empresas servitizadas. Fuente: elaboración propia

\begin{tabular}{cc}
\hline $\mathbf{N} .^{{ }^{\circ} \text { de servicios ofrecidos }}$ & Porcentaje (\%) \\
\hline Oferta de 1 servicio & 25 \\
\hline Oferta de 2 servicios & 7,97 \\
\hline Oferta de 3 servicios & 4,79 \\
\hline Oferta de 4 servicios & 2,28 \\
\hline & 0,86 \\
\hline
\end{tabular}

Este análisis, como panorámica global de la servitización de las empresas españolas, hace aflorar los servicios más ofertados en España, así como aquellos que se ofrecen en menor grado y que, por tanto, constituyen una oportunidad de desarrollo para aquellas compañías que quieran ofrecer servicios diferenciados y novedosos en el mercado. Así, por ejemplo, la instalación e implementación, el transporte o los servicios financieros pueden constituir servicios adicionales de valor añadido que pueden ofrecer las empresas como complemento a sus productos.

Una vez realizada esta descripción inicial sobre la situación de las empresas manufactureras españolas, se ha querido dar un paso más para conocer el efecto de la servitización sobre el rendimiento empresarial, objetivo último de este trabajo. En este sentido, se diseña un análisis de regresión lineal a partir de las variables que se describen a continuación.

\subsection{Variables}

La descripción detallada de las variables consideradas para capturar el efecto de la servitización sobre el rendimiento empresarial son la que recoge la Tabla 4: variables independientes, dependientes y de control.

\subsubsection{Variables dependientes}

El rendimiento empresarial se mide a partir de varios indicadores financieros y económicos. En este trabajo se emplean cuatro de los indicadores más frecuentemente utilizados en la literatura, aceptados por diferentes investigadores como Gebauer (2007), Neely (2008), Suarez et al. (2013), Gebauer y Fleisch (2007), Kwan y Kim (2016), Visintin y Rapaccini (2010) o Visnjic et al. (2016). Estas variables son:
1) ingresos totales de la empresa,

2) beneficio antes de intereses e impuestos (EBIT),

3) rentabilidad sobre las ventas (ROS),

4) beneficio antes de intereses, impuestos, depreciaciones y amortizaciones (EBITDA).

\subsubsection{Variables independientes}

Debido a la innata complejidad de los servicios, es difícil encontrar un indicador preciso y mesurable para la servitización que sea universalmente aplicable. Además, muy pocas empresas se han transformado completamente y han pasado de la oferta única de productos a ser soluciones completas de venta, puesto que la mayoría de ellas están todavía en el proceso incipiente de incorporación de servicios en su cartera de productos.

En este trabajo se utilizan dos formas de medir el efecto de la servitización. En primer lugar, se construye una variable dicotómica (servitización) que toma el valor 1 si las empresas están servitizadas y toma el valor 0 en caso contrario. En este sentido se opta, tal como se describe en el apartado anterior, por considerar en la categoría de servitizadas aquellas empresas que ofrecen al menos uno de los servicios establecidos por Neely (2008). Y, en segundo lugar, se diseña también una variable categórica (servicios) que indica la cantidad de servicios ofrecidos, tomando el valor de 1 a 5 en función de la oferta. Estas mismas variables se utilizan también en los trabajos de Neely (2008) y Chen (2010). 


\subsubsection{Variables de Control}

Con el fin de incluir la influencia de factores que pueden incidir en el rendimiento empresarial se utilizan dos variables de control:

1) tamaño de las empresas medido por el número de empleados (numple),

Tabla 4 Descripción de las variables. Fuente: elaboración propia

\begin{tabular}{llc}
\multicolumn{1}{c}{ Variables } & Descripción & Fuente \\
\hline Dependientes & Ingresos totales no inferiores a 50.000€. & SABI \\
\hline Ingresos & Beneficio antes de intereses e impuestos. & SABI \\
\hline EBIT & Rentabilidad total de la empresa sobre & SABI \\
\hline ROS & las ventas. & SABI \\
\hline EBITDA & $\begin{array}{l}\text { Beneficio antes de intereses, impuestos, } \\
\text { depreciaciones y amortizaciones. }\end{array}$ \\
\hline Independientes & & \\
\hline
\end{tabular}

\begin{tabular}{lll}
\hline Servitización & $\begin{array}{l}\text { Variable dicotómica, que toma el valor } 1 \\
\text { si las empresas están servitizadas, o 0 en } \\
\text { caso contrario. }\end{array}$ & Web de las empresas y FACTIVA \\
\hline Servicios & $\begin{array}{l}\text { Variable categórica que toma el valor de } \\
1 \text { a } 5 \text { según el número de servicios ofre- } \\
\text { cidos. }\end{array}$ & Web de las empresas y FACTIVA \\
\hline De control & Tamaño de las empresas medido por el \\
\hline número de empleados (Numple) & SuBm de empleados \\
\hline Endeudamiento & $\begin{array}{l}\text { Ratio financiera que mide la relación } \\
\text { existente entre el importe de los fondos } \\
\text { propios de una empresa con relación a las } \\
\text { deudas que mantiene tanto a largo como } \\
\text { a corto plazo. }\end{array}$ \\
\hline
\end{tabular}

La Tabla 5 resume los estadísticos descriptivos como el número de observaciones válidas, media, desviación estándar o valor mínimo y máximo de las variables diseñadas.
2) ratio activo-pasivo (endeudamiento).

Estas variables se utilizan también en estudios previos como los de Kwak y Kim (2016) o Li et al. (2015). 
Tabla 5 Estadísticos resumidos.

\begin{tabular}{lccccc}
\hline \multicolumn{1}{c}{ Variable } & Observaciones & Mean & Std. Dev & Min & Max \\
\hline Ingresos & 927 & $328,274.10$ & $1,210,420.00$ & 6,4185 & $2.25 \mathrm{e}+07$ \\
\hline $\begin{array}{l}\text { Número de em- } \\
\text { pleados }\end{array}$ & 927 & 568.20 & 1051.58 & 1 & 12,671 \\
\hline Endeudamiento & 927 & 58.89 & 33.09 & 0.967 & 661.8 \\
\hline ROS & 927 & 5.65 & 19.54 & -83.552 & 485.3 \\
\hline EBITDA & 927 & $19,799.14$ & 58370.89 & $-39,676.00$ & $776,067.8$ \\
\hline EBIT & 927 & $11,166.87$ & 56555.5 & $-754,032.00$ & $771,426.9$ \\
\hline Ln (Ingresos)* & 927 & 18.86 & 0.87 & 17.97 & 23.8 \\
\hline
\end{tabular}

Ln=Logaritmo neperiano

* Se utiliza el logaritmo neperiano de los ingresos para obtener una distribución más simétrica.

\subsection{Análisis empírico}

Para validar si la servitización afecta de manera significativa a las diferentes medidas del rendimiento empresarial, se realiza un análisis de regresión lineal. Debido a la naturaleza continua de las variables dependientes, se considera que el método de Mínimos Cuadrados Ordinarios (OLS) es el más idóneo para este análisis. En este sentido, se consideran dos modelos EQ (1) y EQ (2), ya validados por los trabajos anteriores de Neely (2008) y Chen (2010).

Las ecuaciones de los modelos son:

$y=\propto+\beta \_1$ SERVITIZACIÓN $+\beta \_2$ NUMPLE +

$+\beta \_3$ Endeudamiento $+\varepsilon$

$\mathrm{y}=\theta+\beta \_1$ SERVICIOS $+\beta \_2$ NUMPLE +

$+\beta \_3$ Endeudamiento $+u$

\section{Resultados}

La Tabla 6 presenta los resultados de la estimación de la EQ(1) para las cuatro medidas de rendimiento empresarial (Ingresos, EBIT, ROS, EBITDA). Estos resultados muestran un efecto negativo y estadísticamente significativo de la estrategia de servitización sobre el rendimiento empresarial. Esto parece ir en la línea de otros estudios recientes, como el de Neely (2008), que sugiere una influencia negativa explicada porque la estrategia de la servitización representa un gran reto que requiere nuevos procesos y estructuras para su implementación.

Con relación a las variables de control se obtiene el efecto esperado. El tamaño de la empresa tiene una influencia positiva y estadísticamente significativa en relación a los indicadores de rendimiento empresarial, con excepción de la variable ROS. Una explicación de este efecto positivo se debe a las economías de escala que aprovechan las grandes empresas. En el caso de la variable endeudamiento, no se encuentra significancia estadística en su relación con el rendimiento de la empresa. 
Tabla 6 Impacto de la servitización en el rendimiento empresarial.

\begin{tabular}{|c|c|c|c|c|c|}
\hline & (1) & (2) & (3) & (4) & (5) \\
\hline VARIABLES & Ingresos $(€)$ & EBIT $(€)$ & ROS (\%) & EBITDA $(€)$ & $\begin{array}{l}\text { Logaritmo nepe- } \\
\text { riano de ingresos }\end{array}$ \\
\hline Servitización & $3.432 \mathrm{e}+7$ & $-7,378.953^{*}$ & $-3.021 * *$ & $-6,938.451^{*}$ & $-0.200 * * *$ \\
\hline \multirow{3}{*}{$\begin{array}{l}\text { Número de em- } \\
\text { pleados }\end{array}$} & $(7.211 \mathrm{e}+7)$ & $(3,807.973)$ & (1.319) & $(3,681.923)$ & $(0.046)$ \\
\hline & $5.422 \mathrm{e}+5 * * *$ & $4.507 * *$ & -0.000 & $20.452 * * *$ & $0.001^{* * *}$ \\
\hline & $(3.381 \mathrm{e}+5)$ & (1.786) & $(0.001)$ & $(1.727)$ & $(0.000)$ \\
\hline \multirow[t]{2}{*}{ Endeudamiento } & $7.28 \mathrm{e}+5$ & -80.539 & 0.019 & -70.026 & 0.001 \\
\hline & $(1.061 \mathrm{e}+6)$ & (56.064) & (0.019) & (54.208) & $(0.001)$ \\
\hline \multirow[t]{2}{*}{ Constante } & $-3.683 e+07$ & $16,368.024 * * *$ & $5.827 * * *$ & $15,140.265$ & $18.603^{* * *}$ \\
\hline & $(7.798 \mathrm{e}+7)$ & $(4,118.044)$ & (1.426) & $(3,981.730)$ & $(0.050)$ \\
\hline Observations & 927 & 927 & 927 & 927 & 927 \\
\hline R-squared & 0.226 & 0.011 & 0.007 & 0.132 & 0.401 \\
\hline
\end{tabular}

Standard errors in parentheses. *** $\mathrm{p}<0.01$, ** $\mathrm{p}<0.05, * \mathrm{p}<0.1$
A la vista de la relación negativa entre la servitización y el rendimiento empresarial (conjunto de las cuatro variables analizadas: Ingresos, EBIT, ROS, EBITDA)) y, teniendo en cuenta estudios previos que sugieren que esta relación varía en función del número de servicios ofrecidos por la empresa, se ha optado en segundo lugar por realizar un análisis complementario al respecto.

En la Tabla 7 se exponen los resultados obtenidos a partir de la estimación de la EQ(2) para las cuatro medidas del rendimiento empresarial. En este modelo, a través de la variable categórica servicios, se recoge el efecto cuando la empresa ofrece dos, tres, cuatro o cinco servicios, en comparación con aquellas otras que ofrecen un único servicio (constante).

Cuando se oferta un servicio, el efecto de la servitización es positivo y estadísticamente significativo sobre el rendimiento empresarial. Esto apoya la evidencia de que la estrategia de servitización resulta beneficiosa para la empresa porque está relacionada con la rentabilidad sobre sus recursos propios.

Asimismo, se observa que las empresas que están servitizadas con dos servicios presentan un efecto negativo sobre el rendimiento de la empresa en comparación con aquellas con un solo servicio, ya sea medido en términos de Ingresos, EBIT, ROS o EBITDA. No obstante, no se encontró significancia estadística.
Cuando se ofrecen tres servicios el resultado es análogo al caso anterior de la oferta de dos servicios. Sin embargo, sobre la variable Ingresos tiene un efecto positivo y estadísticamente significativo. Es decir, cuando se ofertan tres servicios la empresa aumenta sus ingresos. La reducción de la rentabilidad podría explicarse por la mayor inversión necesaria que implica la apuesta por una oferta superior de servicios.

En cambio, cuando una empresa ofrece cuatro y cinco servicios el efecto es positivo sobre el rendimiento empresarial, de la misma manera que aquellas que ofrecen un único servicio. No obstante, no se encontró significancia estadística para la oferta de cuatro servicios. En el caso de los cinco servicios, su efecto resultó ser estadísticamente significativo sobre los Ingresos, ROS y EBITDA. Como se observa en la Tabla 7, la variable ROS de las empresas servitizadas aumenta significativamente hasta un $7.6 \%$ cuando las empresas ofrecen cinco servicios.

De estos resultados se puede destacar que la cantidad de servicios se relaciona positivamente con los ingresos de una empresa, principalmente en aquellas empresas que ofrecen tres y cinco servicios, en comparación con aquellas que ofertan un único servicio. Esto se puede explicar dado que para la oferta de un solo servicio se requiere proporcionalmente de mayor inversión en recursos humanos para promover la servitización. 
Finalmente, el efecto de las variables de control (número de empleados y endeudamiento) muestran el mismo comportamiento conforme lo obtenido anteriormente en la Tabla 5. Existe una correlación positiva entre el tamaño de la em- presa y las diferentes medidas del rendimiento de la empresa (ingresos totales de la empresa, EBI, ROS y EBITDA), por lo que cuanto más grande sea la empresa (medida por el número de empleados) mayores serán sus ingresos.
Tabla 7 Efecto del número de servicios sobre la servitización.

\begin{tabular}{|c|c|c|c|c|c|}
\hline & (1) & (2) & (3) & (4) & (5) \\
\hline VARIABLES & $\begin{array}{c}\text { Últimos ingresos } \\
(€)\end{array}$ & EBIT $(€)$ & $\begin{array}{c}\text { Rentabilida d } \\
\text { económica }(\%)\end{array}$ & EBITDA $(€)$ & $\begin{array}{l}\text { Logaritmo nepe- } \\
\text { riano de ingresos }\end{array}$ \\
\hline \multirow[t]{2}{*}{ Oferta 2 Servicios } & $-1.220 \mathrm{e}+08$ & -400.4 & -2.488 & $-2,714.7$ & $-0.161^{*}$ \\
\hline & $(1.285 \mathrm{e}+08)$ & $(6,850.8)$ & (2.384) & $(6,625.8)$ & $(0.083)$ \\
\hline \multirow[t]{2}{*}{ Oferta 3 Servicios } & $7.981 \mathrm{e}+08 * * *$ & $-26,193.8 * * *$ & -4.534 & $-8,323.8$ & $0.283^{* * *}$ \\
\hline & $(1.666 \mathrm{e}+08)$ & $(8,882.4)$ & (3.091) & $(8,590.7)$ & $(0.108)$ \\
\hline \multirow[t]{2}{*}{ Oferta 4 Servicios } & $1.808 \mathrm{e}+08$ & $4,429.8$ & 3.637 & $13,877.6$ & 0.163 \\
\hline & $(2.544 \mathrm{e}+08)$ & $(13,560.4)$ & (4.719) & $(13,115.1)$ & $(0.164)$ \\
\hline \multirow[t]{2}{*}{ Oferta 5 Servicios } & $3.121 \mathrm{e}+08$ & $23,640.2$ & $7.663 * * *$ & $52,997.4 * *$ & $0.468 *$ \\
\hline & $(4.075 \mathrm{e}+08)$ & $(21,722.4)$ & (7.559) & $(21,009.1)$ & $(0.263)$ \\
\hline \multirow{2}{*}{$\begin{array}{l}\text { Número de emplea- } \\
\text { dos }\end{array}$} & $5.0309 \mathrm{E} 5 * * *$ & $4.2^{* *}$ & 0.000 & $19.2^{* * *}$ & $0.000^{* * *}$ \\
\hline & $(39,010.825)$ & $(2.080)$ & $(0.001)$ & $(2.011)$ & $(0.000)$ \\
\hline \multirow[t]{2}{*}{ Endeudamiento } & $649,467.760$ & -74.126 & 0.020 & -64.7 & 0.001 \\
\hline & (1050162.677) & (55.983) & $(0.019)$ & $(54.1)$ & $(0.001)$ \\
\hline \multirow[t]{2}{*}{ Constante } & $-3.072 \mathrm{e}+07$ & $14,298.7 * * *$ & $4.920 * * *$ & $13,136.5^{* * *}$ & $18.535^{* * *}$ \\
\hline & $(72831317.050)$ & $(3,882.5)$ & $(1.351)$ & $(3,755.1)$ & $(0.047)$ \\
\hline Observations & 927 & 927 & 927 & 927 & 927 \\
\hline R-squared & 0.246 & 0.019 & 0.005 & 0.138 & 0.398 \\
\hline
\end{tabular}

Standard errors in parentheses ${ }^{* * *} \mathrm{p}<0.01,{ }^{* *} \mathrm{p}<0.05,{ }^{*} \mathrm{p}<0.1$ 


\section{Conclusiones}

Aunque la literatura previa ha enfatizado en la importancia de la implementación de la estrategia de servitización, todavía existe un largo recorrido por abordar por parte de las empresas españolas. Un dato revelador en este sentido es que, en España, para la muestra analizada, menos de la mitad de las empresas (41\%) incluyen el proceso de servitización dentro de su estrategia empresarial. Así mismo, De la Calle y Freije (2016) confirman el interés de las empresas manufactureras españolas por intentar el proceso de servitización en algún momento. De este modo, tal como ya anticiparon algunas investigaciones en otros países, como los trabajos de Gebauer et al. (2005;2012), Neely (2008) o Crozet y Millet (2017), se demuestra también en el caso español que la adopción de la servitización es un proceso lento en las empresas. Ello abre una futura línea de investigación sobre el análisis de rentabilizar la estrategia de servitización a largo plazo.

Este es un dato en el cual las empresas españolas pueden apoyarse para pensar en una vía alternativa e incluir la estrategia de servitización en sus empresas, ya que mediante la expansión del modelo de negocio y la inclusión de los servicios, los fabricantes tradicionales llegan a ofrecer soluciones cada vez más valoradas por los clientes.

No obstante, hasta ahora el proceso de servitización es lento debido al vacío de información para recomendar como implementar la estrategia de servicio, además de la complejidad que conlleva este proceso y la inversión. Este estudio demuestra como deberían de implementarse correctamente la cantidad de servicio para que tener un impacto positivo.

Según la muestra estudiada de empresas servitizadas en España, a través del rendimiento empresarial, se concluye que ofrecer servicios no es sinónimo de éxito. Ello coincide con la literatura previa según la cual una estrategia exitosa requiere también de una alineación adecuada del entorno, la estrategia y los factores de diseño organizacional (por ejemplo, Gebauer et al. (2005), Neu y Brown (2005) o Gebauer et al. (2007)).

Este trabajo demuestra que, al separar las variables por la cantidad de servicios ofrecidos, cuando existen tres o cinco servicios ofrecidos por las empresas, el rendimiento empresarial es positivo en función de los ingresos totales de la empresa (ingresos brutos) en comparación con aquellas que ofrecen un único servicio, en la línea de estudios anteriores como el trabajo de Li et al. (2015) o De la calle y Freije (2016).

Asimismo, la rentabilidad de las empresas incrementa a medida que los servicios aumentan (cinco servicios) con una rentabilidad de hasta un $7 \%$. Por otro lado, el número de empleados o tamaño de la empresa no afecta a la rentabilidad significativamente, pero sí fuertemente en el rendimiento financiero de la empresa.
La implementación de la estrategia de servitización en el modelo de negocio debe coincidir con una progresión controlada, ya que ofrecer más servicios tampoco es garantía de incrementar los ingresos totales. En este sentido, encontramos que cuando se ofrece un servicio, el efecto es positivo sobre el rendimiento empresarial, pero cuando se ofertan dos $\mathrm{y}$ tres servicios, no es tan beneficioso.

Por último, cabe destacar que algunos autores han identificado la necesidad de llegar a una mínima masa crítica para conseguir obtener el impacto económico en la estrategia de servitización. Fang et al. (2008) consideran que la incidencia de la transición de una empresa de fabricación para añadir servicios de valor es nula o incluso negativa hasta que la firma alcanza una masa crítica de ventas de servicios alrededor de un $20 \%$ - 30\%, después de lo cual tiene un efecto cada vez más positivo.

Lo que sí parece indiscutible es la dificultad de la implementación de la estrategia de servitización. De hecho, los servicios no son por sí mismos garantía de rápidos resultados. El cuidado de la implementación de la estrategia es imprescindible.

En consecuencia, los casos de éxito justifican el interés de una mayor investigación sobre las condiciones específicas de implementación de la servitización, ya que lo que se evidencia según los datos analizados, es su dificultad para traducirlo en resultados empresariales. En este sentido, de acuerdo con de la Calle y Freije (2016), es interesante plantear como futura línea de investigación el análisis del tipo concreto de servicios ofertados distinguiendo su naturaleza y su conexión, enlace o vínculo con la estrategia general de la empresa, si son principalmente instrumentales para apoyar el negocio tradicional o si, por el contrario, éstos abren nuevas oportunidades de negocio, también, por ejemplo, un análisis por provincias que podría resultar de gran utilidad para añadir nuevos argumentos y reforzar las conclusiones obtenidas y comparar los resultados a nivel más local.

\section{Bibliografía}

Arias-Aranda, D., and Jaría-Chacón, N. (2014), “Servitization and value creation: deploying to mobile device platforms”, Dyna, Vol. 89 No.5, pp. 482.

Baines, T., Lightfoot, H., Peppard, J., Johnson, M., Tiwari, A., Shehab, Essam., and Swink, M. (2009), "Towards an operations strategy for product-centric servitization”, International Journal of Operations \& Production Management, Vol. 29 No. 5, pp. 494-519.

Baines, T., Lightfoot, H. and Smart, P. (2011), "Servitization within manufacturing Exploring the provision of advanced services and their impact on vertical integration”, Journal of Manufacturing Technology Management, Vol. 22 No. 7, pp. 947-954. 
Baines, T.S., Lightfoot, H.W., Evans, S., Neely, A., Greenough, R., Peppard, J., Roy, R., Shehab, E., Braganza, A., Tiwari, A., Alcock, J.R., Angus, J.P., Bastl, M., Cousens, A., Irving, P., Johnson, M., Kingston, J., Lockett, H., Martinez, V., Michele, P., Tranfield, D., Walton, I.M. and Wilson, H. (2007), "State-of-the-art in product-service systems", Proceedings of the Institution of Mechanical Engineers, Part B: Journal of Engineering Manufacture, Vol. 221 No. 10, pp. 1543-1552

Baines, T. and Lightfoot, H. (2013), Made to Serve. What it takes for a Manufacturer to Compete

Through Servitization and Product-Service Systems, Wiley, Chichester.

Brady, T., Davies, A. and Gann, D.M. (2005), "Creating value by delivering integrated solutions”, International Journal of Project Management, Vol. 23 No. 5, pp. 360365.

Chen, J. X., (2010), “An empirical test of the effect of manufacturing service-orientation on corporate performance: A comparison between Chinese and American enterprises”, Journal of Business Economics, Vol. 4, pp. 33-41.

Dachs, B., Biege, S., Borowiecki, M., Lay, G., Jäger, A. and Schartinger, D. (2014), "Servitisation in European manufacturing industries: empirical evidence from a largescale database”, The Service Industries Journal, Vol. 34 No. 1, pp. 5-23.

Davies, A. (2004), "Moving base into high-value integrated solutions: a value stream approach”, Industrial and Corporate Change, Vol. 13, No.5, pp. 727-756.

Davies, A., Brady, T. and Hobday, M. (2007), “Organizing for solutions: system seller vs systems integrator”, Industrial Marketing Management, Vol. 36 No. 2, pp. 183193.

De la Calle and Freije, I. (2016): “Is servitization really profitable? Two decades of evidence from Spanish manufacturing companies”, Universia Business Review, No. 49, first quarter, pp. 54-95.

Eggert, A., Hogreve, J., Ulaga, W. and Muenkhoff, E. (2011), "Industrial services, product innovations, and firm profitability: a multiple-group latent growth curve analysis”, Industrial Marketing Management, Vol. 40 No. 5, pp. 661-670.

Eggert, A., J. Hogreve, W. Ulaga, and E. Muenkhoff. (2014), "Revenue and profit implications of industrial service strategies", Journal of Service Research Vol.17 No.1, pp. 23-39.
Falk, M. (2014), “The impact of new goods and service products on firm growth: evidence from Austrian-linked firm-level data", Economics of Innovation and New Technology, Vol. 23 No.4, p. 378-397.

Fang, E., Palmatier, R.W., and Steenkamp, J.E.M. (2008), "Effect of service transition strategies on firm value", Journal of Marketing, Vol. 72 No.9, pp. 1-14.

Fiksdal, I. and Kumar, K., (2011), Servitization in Norwegian. Master's Thesis, Norwegian University of Science and Technology.

Fischer, T., Gebauer, H., Gregory, M., Ren, G. and Fleisch, E. (2010), "Exploitation or exploration in service business development?: Insights from a dynamic capabilities perspective”, Journal of Service Management, Vol. 21 No. 5, pp. 591-624.

Gebauer, H. (2007), “An investigation of antecedents for the development of customer support services in manufacturing companies”, Journal of Business-to-Business Marketing, Vol. 14 No.3, pp. 59-96.

Gebauer, H. Fleish, E. and Friedli, T. (2005), “ Overcoming the service paradox in manufacturing companies”, European Management Journal, Vol. 23 No. 1, pp. 14-26.

Gebauer , H., Friedli, T. and Fleisch , E., (2006), “Success factors for achieving high service revenues in manufacturing companies”, Benchmarking: An International Journal, Vol. 13 No.3, pp. 374-386.

Gebauer, H. and Fleisch, E. (2007), “An investigation of the relationship between behavioural processes, motivation, investments in the service business and service revenue”, Industrial Marketing Management, Vol. 36 No. 3, pp. 337-348.

Gebauer, H., Edvardsson, B., Gustafsson, A. and Witell, L. (2010), "Match or Mismatch: Strategy-Structure Configurations in the Service Business of Manufacturing Companies”, Journal of Service Research, Vol. 13 No. 2, pp. 198-215.

Gebauer, H., Bravo-Sanchez, C. and Fleisch, E. (2008), "Service strategies in product manufacturing companies”, Business Strategy Series, Vol. 9 No. 1, pp. 12 - 20.

Gebauer, H., Ren, G.-J., Valtakoski, A. and Reynoso, J. (2012), "Service-driven manufacturing: provision, evolution and financial impact of services in industrial firms", Journal of Service Management, Vol. 23 No. 1, pp. 120-136. 
Gujarati, Damodar N. and Porter, Dawn. (2010), Econometría (4 a ed.), McGraw Hill.

Han, S., Kuruzovich, J. and Ravichandran, T. (2013), "Service expansion of product firms in the information technology industry: An empirical study”, Journal Management Information System, Vol. 29 No. 4 pp. 127-158.

Homburg, C., Fassnacht, M. and Guenther, C. (2003), “The role of soft factors in implementing a service-oriented strategy in industrial marketing companies”, Journal of Business-to Business Marketing, Vol. 10 No. 2, pp. 2351.

Kohtamäki, M., Partanen, J., Parida, V. and Wincent, J. (2013), “ Non-linear relationship between industrial service offering and sales growth: the moderating role of network capabilities”, Industrial Marketing Management, Vol. 42 No. 8, pp. 1374-1385.

Kowalkowski, C. (2010). What does a service-dominant logic really mean for manufacturing firms? CIRP Journal of Manufacturing Science and Technology, 3(4), 285-292.

Kwak, K \& Kim, W. (2016), "Effect of service integration strategy on industrial firm performance", Journal of Service Management, Vol. 27 No.3, pp. 391-430.

Li, J. H., Lin, L., Chen, D. P. \& Ma, L. Y. (2015), “An empirical study of servitization paradox in China”, Journal of High Technology Management Research, Vol. 26 No. 1, p. 66-76.

Malleret , V. (2006), "Value Creation through service offers”, European Management Journal, Vol. 24, No. 1, pp. 106-116 .

Meier, H., Völker, O., and Funke, B. (2011), “Industrial product-service system (IPS2): Paradigm shift by mutually determined products and services”, International Journal of Advance Manufacturing Technology, Vol. 59, No. 9-12, pp. 1175-1191.

Martinez, V., Bastl, M., Kingston, J. and Evans, S., (2010), "Challenges in transforming manufacturing organisations into product-service providers”, Journal of Manufacturing Technology Management, Vol. 21 No. 4, pp. 449-469..

Neely, A. (2008), "Exploring the financial Consequences of the servitization of manufacturing”, Operations Management Research, Vol. 1 No. 2, pp. 103-118.
Neely, A., Benedettini, O. and Visnjic, I., (2011), “The servitization of manufacturing: Further evidence”, paper presented at the EuOMA Conference, Cambridge, 3-6 July, available at: www.cambridgeservicealliance.org/ outputs/papers.html (accessed 11 October, 2014).

Neu, W. and Brown, S. (2005), "Forming Successful Business-to-Business Services in Goods-Dominant Firms”, Journal of Service Research, Vol. 81, No.1, pp. 3-17.

Nordin, F., Kindström, D., Kowalkowski, C., And Rehme, J. (2011), “The risks of providing services: Differential risk effects of the service-development strategies of customisation, bundling, and range”, Journal of Service Management, Vol. 22 No.3, pp. 390-408.

Oliva, R. and Kallenberg, R. (2003), "Managing the transition from products to services" International Journal of Service Industry Managemente, Vol. 14 No. 2, pp. 160172.

Ostrom, A., Bitner, M., Brown, S., Burkhard, K., Goul, M., Smith-Daniels., V. Demirkan, H., and Rabinovich, E. (2010), "Moving forward and making a difference: Research priorities for the science of service”, Journal of Service Research, Vol. 13 No. 1, pp. 4-36.

Potts, G. W. (1988), "Exploiting your product's life cycle”, Harvard Business Review, Vol. 66 No.5, pp. 32-35.

Reinartz, W. and Ulaga, W. (2008), "How to sell services profitably", Harvard Business Review, Vol. 86 No. 5, pp. 90-96.

Slack, N. (2005), “Operations strategy: will it ever realise its potential?”, Gestão \& Produção, Vol. 12 No. 3, pp. 323-332.

Salonen, A. (2011), "Service transition strategies of industrial manufacturers”, Industrial Marketing Management, Vol. 40 No. 5, pp. 683-690.

Suarez, F. F., Cusumano, M. and Kahl, S.J. (2013), “Services and the business models of product firms: an empirical analysis of the software industry”, Management Science, Vol. 59 No.2, pp. 705-716.

Slepniov, D., Waehrens, . V. B. and Johansen, J. (2010), "Servitization as a strategy for survival: An investigation of the process in danish manufacturing firms". Paper for the 15th Cambridge International Manufacturing Symposium. Aalborg, Center for Industrial Production: 1-12., s.n. 
Vandermerwe, S. and Rada, J., (1988), "Servitization of business: adding value by adding services”, European Management Journal, Vol. 6 No. 4, pp. 314-324.

Visnjic Kastalli, I. and Van Looy, B., 2013, "Servitization: Disentangling the impact of service business model innovation on manufacturing firm performance", Journal of Operations Management, Vol. 31, pp. 169-180.
Visintin F. and Rapaccini M. (2010), "Exploring the linkage between servitization and financial performances: evidences from the HVAC industry”, Proceedings of APMS 2010 - International Conference on Advances in Production Management Systems.

Wise, R. and Baumgarther, P. (1999), “Go downstream”. Harrvard Business Review, Vol. 77 No. 5, pp. 133-141. 